\title{
Evaluation of appropriate follow-up after curative surgery for patients with colorectal cancer using time to recurrence and survival after recurrence: a retrospective multicenter study
}

\begin{abstract}
Tomoki Yamano ${ }^{1}$, Shinichi Yamauchi ${ }^{2}$, Kiyoshi Tsukamoto ${ }^{1}$, Masafumi Noda $^{1}$, Masayoshi Kobayashi ${ }^{1}$, Michiko Hamanaka ${ }^{1}$, Akihito Babaya ${ }^{1}$, Kei Kimura ${ }^{1}$, Chihyon Son ${ }^{1}$, Ayako Imada ${ }^{1}$, Shino Tanaka ${ }^{1}$, Masataka Ikeda ${ }^{1}$, Naohiro Tomita ${ }^{1}$, Kenichi Sugihara $^{2}$ and Japanese Study Group for Postoperative Follow-up of Colorectal Cancer

${ }^{1}$ Division of Lower GI Surgery, Department of Surgery, Hyogo College of Medicine, Hyogo, Japan

${ }^{2}$ Division of Colorectal Surgery, Department of Surgery, Tokyo Medical and Dental University, Tokyo, Japan

Correspondence to: Tomoki Yamano, email: yamanot@hyo-med.ac.jp

Keywords: colorectal cancer; curative surgery; follow-up; recurrence; survival

Received: March 02, $2018 \quad$ Accepted: April 12, $2018 \quad$ Published: May 22, 2018

Copyright: Yamano et al. This is an open-access article distributed under the terms of the Creative Commons Attribution License 3.0 (CC BY 3.0), which permits unrestricted use, distribution, and reproduction in any medium, provided the original author and source are credited.
\end{abstract}

\section{ABSTRACT}

Introduction: The follow-up schedule for colorectal cancer patients after curative surgery is inconsistent among the guidelines. Evaluation of time to recurrence (TTR) and survival after recurrence (SAR) may provide evidence for appropriate follow-up.

Methods: We assessed 3039 colon cancer (CC) and 1953 rectal cancer (RC) patients who underwent curative surgery between 2007 and 2008. We evaluated the pre- and post-recurrent clinicopathological factors associated with TTR and SAR in each stage of $\mathrm{CC}$ and $\mathrm{RC}$.

Results: The recurrence rates of stages I, II, and III were $1.2 \%, 13.1 \%$, and

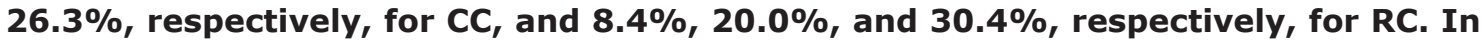
CC patients, high carcinoembryonic antigen (CEA) level and lymphovascular invasion were independent predictors of short TTR. In RC patients, metastatic factors (liver metastasis in stage III) and venous invasion (stage III) were independent predictors of short TTR. The prognostic factors of SAR were age (stage II CC and stage III RC), female gender (stage III RC), high CEA level (stage II RC), histological type (stage III CRC), nodal status (stage III CC), recurrence within 1 year (stage III RC), M1b recurrence (stage II CRC), local recurrence (stage II CC), and no surgical resection after recurrence (stage II and III CRC).

Conclusions: The follow-up schedule for stage I should be different from that for the other stages. We recommend that intensive follow-up is appropriate in stage III CC patients with undifferentiated adenocarcinoma or N2 nodal status, stage II RC patients with high preoperative CEA level, and stage III RC patients.

\section{INTRODUCTION}

Colorectal cancer (CRC) is one of the most common malignancies worldwide [1]. CRC has been increasing annually in Japan, and was estimated to be the most common malignancy in 2016 and the second most common cause of cancer-related deaths in 2014 [2].
Recurrence rates vary among patients who receive curative surgery, with recurrence reported in about 30\% of stage III patients, $15 \%$ of stage II patients, and $<5 \%$ of stage I patients in Japan [3]. Adequate surveillance is therefore required to both reduce costs and improve survival. However, surveillance is generally provided for patients without any recurrence. 
The usefulness of intensive surveillance with routine carcinoembryonic antigen (CEA) monitoring and computed tomography (CT) scans remains controversial. Although early detection of recurrence has been shown to improve survival, early detection using intensive surveillance did not always result in improved overall survival [4-9]. The guidelines of the Japanese Society for Cancer of the Colon and Rectum propose intensive surveillance, including CEA checks every 3 months and CT every 6 months for 3 years, regardless of clinical stage and risk of recurrence [10]. This reflects the intensive surveillance schedules recommended by the European Society for Medical Oncology, American Society of Clinical Oncology, and National Comprehensive Cancer Network (NCCN) guidelines, which recommended CEA checks every 3-6 months and CT every 6-12 months for $2-3$ years, depending on the risk of the patient [11-15].

However, these surveillances are not strictly categorized by risk factors other than clinical stage. Information on time to recurrence (TTR) and survival after recurrence (SAR) may help to determine the appropriate follow-up schedule, and has previously been used to evaluate the usefulness of intensive follow-up in clinical trials and meta-analysis $[3,5,7,8]$.

In this study, we evaluated the clinicopathological factors predicting TTR and SAR in patients with colon cancer (CC) or rectal cancer (RC) during intensive follow-up after curative resection, to determine the factors influencing the appropriate follow-up schedule.

\section{RESULTS}

\section{Differences in clinicopathological factors between $\mathrm{CC}$ and $\mathrm{RC}$}

We compared the clinicopathological factors between $\mathrm{CC}$ and $\mathrm{RC}$ (Table 1). Age group, gender, preoperative CEA level, tumor depth, nodal status, venous invasion, application of adjuvant therapy, and clinical stage all differed significantly between the two locations.

\section{Factors associated with recurrence}

The recurrence rates of stages I, II, and III CC were $1.2 \%(11 / 890), 13.1 \%(151 / 1149)$, and $26.3 \%$ (263/1000), and of RC were 8.4\% (54/641), 20.0\% (123/614), and 30.4\% (212/697), respectively (Table 1). High preoperative CEA level, tumor depth, nodal status, lymphatic invasion, venous invasion, and clinical stage were significantly associated with recurrence, regardless of tumor location (Table 1). Gender and histological type were significantly associated with recurrence in $\mathrm{RC}$, but not in CC.

We also assessed the influence of tumor location on recurrence in relation to each clinicopathological factor (Table 1). There were significant differences in recurrence rates between $\mathrm{CC}$ and $\mathrm{RC}$ for patients in each age group, with each CEA level, each histological type, and with tumor depth of T1-3, N0 nodal status, lymphatic invasion of $1 \mathrm{y} 0-2$, venous invasion of $\mathrm{v} 0-2$, stage I-II and stage I-II without adjuvant therapy. However, there were no significant differences in recurrence rates by tumor location in relation to clinically-advanced factors including: depth of T4, N1 and N2 nodal status, lymphatic invasion of ly3, venous invasion of v3, and stage III.

\section{Factors associated with RFS and OS}

RFS was significantly influenced by preoperative CEA level, histological type, tumor depth, nodal status, stage, lymphatic invasion, and venous invasion, regardless of tumor location (Figures 1, 2). RFS was also significantly influenced by gender in patients with RC (Figure 2). OS was significantly influenced by age, gender, preoperative CEA level, tumor depth, nodal status, stage, lymphatic invasion, and venous invasion, regardless of tumor location (Figures 3, 4). OS was also significantly influenced by histological type in RC alone (Figure 4).

\section{Clinicopathological factors in patients with recurrence}

Age group, gender, tumor depth, nodal status, stage, TTR, type of recurrence, and sites of recurrence differed significantly between $\mathrm{CC}$ and $\mathrm{RC}$ in patients with recurrence (Table 2). The liver was the most common metastatic site in CC patients, followed by the lungs, peritoneum, and distant lymph nodes. In contrast, the lungs were the most common metastatic site in $\mathrm{RC}$ patients, followed by the liver, local recurrence, and distant lymph nodes. There was no significant difference in preoperative CEA, histological type, or treatment after recurrence according to tumor location.

\section{Time to recurrence (TTR)}

TTR was significantly longer in stage III patients with RC compared with CC patients, but not in stage I and II patients (Figure 5A-5C). The 1-, 2-, 3-, and 5 -year accumulative recurrence rates in $\mathrm{CC}$ patients were $18 \%, 46 \%, 73 \%$, and $91 \%$ for stage I; $46 \%, 78 \%$, $90 \%$ and $99 \%$ for stage II; $53 \%, 80 \%, 94 \%$ and $97 \%$ for stage III, respectively (Figure 5D). The equivalent recurrence rates in RC patients were $17 \%, 41 \%, 65 \%$, and $87 \%$ for stage I; $49 \%, 81 \%, 94 \%$, and $98 \%$ for stage II; $44 \%, 71 \%, 83 \%$, and $98 \%$ for stage III respectively (Figure 5E).

We further assessed TTR for CC and RC patients depending on stage, except for stage I, because there were fewer patients and longer TTR than for the other stages.

TTR in stage II CC was significantly associated with gender, preoperative CEA level, lymphatic 
Table 1: Clinicopathological factors of patients by tumor location

\begin{tabular}{|c|c|c|c|c|c|c|c|c|}
\hline \multirow{2}{*}{$\frac{\text { Location }}{\text { Factors }}$} & \multirow{2}{*}{$\begin{array}{c}\mathrm{CC} \\
(\mathrm{N}=3039) \\
\text { Number } \\
(\%)\end{array}$} & \multirow{2}{*}{$\begin{array}{c}\text { RC } \\
(\mathrm{N}=1953) \\
\text { Number } \\
(\%)\end{array}$} & \multirow{2}{*}{$\begin{array}{c}P \text { value } \\
\text { CC vs } \\
\text { RC }\end{array}$} & \multicolumn{2}{|c|}{$\mathbf{C C}$} & \multicolumn{2}{|c|}{$\mathbf{R C}$} & \multirow{2}{*}{$\begin{array}{c}P \text { value } \\
\text { CC vs } \\
\text { RC }\end{array}$} \\
\hline & & & & $\begin{array}{l}\text { Recurrence } \\
\text { rate }\end{array}$ & $P$ value & $\begin{array}{l}\text { Recurrence } \\
\text { rate }\end{array}$ & $P$ value & \\
\hline \multicolumn{9}{|l|}{ Age } \\
\hline$\leq 64$ & $1041(34)$ & $998(51)$ & \multirow{3}{*}{$<0.0001$} & $15.4 \%$ & \multirow{3}{*}{ NS } & $19.3 \%$ & \multirow{3}{*}{ NS } & 0.018 \\
\hline $65-74$ & $1090(36)$ & $629(32)$ & & $13.1 \%$ & & $21.6 \%$ & & $<0.0001$ \\
\hline $75 \leq$ & $908(30)$ & $326(17)$ & & $13.4 \%$ & & $18.4 \%$ & & 0.03 \\
\hline \multicolumn{9}{|l|}{ Gender } \\
\hline Male & $1678(55)$ & $1192(61)$ & \multirow{2}{*}{$<0.0001$} & $14.8 \%$ & \multirow{2}{*}{ NS } & $21.4 \%$ & \multirow{2}{*}{0.041} & $<0.0001$ \\
\hline Female & $1361(45)$ & $761(39)$ & & $13.0 \%$ & & $17.6 \%$ & & 0.0044 \\
\hline \multicolumn{9}{|c|}{ Preoperative CEA } \\
\hline High & $831(27)$ & $561(29)$ & \multirow{3}{*}{0.043} & $23.9 \%$ & \multirow{3}{*}{$<0.0001$} & $30.5 \%$ & \multirow{3}{*}{$<0.0001$} & 0.0068 \\
\hline Normal & $2115(70)$ & $1310(67)$ & & $10.3 \%$ & & $15.3 \%$ & & $<0.0001$ \\
\hline Unknown & 93 ( 3 ) & $82(4)$ & & $9.7 \%$ & & $20.7 \%$ & & 0.04 \\
\hline \multicolumn{9}{|c|}{ Histological Type } \\
\hline $\begin{array}{c}\text { Well/ } \\
\text { Moderately }\end{array}$ & $2867(94)$ & $1881(96)$ & \multirow{3}{*}{0.0069} & $13.6 \%$ & \multirow{3}{*}{ NS } & $19.2 \%$ & 0.0002 & \multirow{3}{*}{$<0.0001$} \\
\hline Poorly & $74(2)$ & $31(2)$ & & $21.6 \%$ & & $41.9 \%$ & 0.034 & \\
\hline Mucinous & $98(3)$ & $41(2)$ & & $18.4 \%$ & & $36.6 \%$ & 0.021 & \\
\hline \multicolumn{9}{|l|}{ Tumor depth } \\
\hline $\mathrm{T} 1$ & $592(19)$ & $355(18)$ & \multirow{4}{*}{$<0.0001$} & $1.0 \%$ & \multirow{4}{*}{$<0.0001$} & $4.8 \%$ & \multirow{4}{*}{$<0.0001$} & 0.0003 \\
\hline $\mathrm{T} 2$ & $447(15)$ & $438(22)$ & & $3.6 \%$ & & $11.9 \%$ & & $<0.0001$ \\
\hline $\mathrm{T} 3$ & $1411(46)$ & $971(50)$ & & $14.0 \%$ & & $25.4 \%$ & & $<0.0001$ \\
\hline $\mathrm{T} 4$ & $589(19)$ & $189(10)$ & & $35.0 \%$ & & $38.6 \%$ & & NS \\
\hline \multicolumn{9}{|l|}{ Nodal status } \\
\hline N0 & $2039(67)$ & $1256(64)$ & \multirow{3}{*}{$<0.0001$} & $7.9 \%$ & & $14.1 \%$ & & $<0.0001$ \\
\hline N1 & $786(26)$ & $483(25)$ & & $22.3 \%$ & $<0.0001$ & $26.3 \%$ & $<0.0001$ & NS \\
\hline $\mathrm{N} 2$ & $214(7)$ & $214(119)$ & & $41.1 \%$ & & $39.7 \%$ & & NS \\
\hline Lymphatic invas & & & & & & & & \\
\hline ly0 & $1323(44)$ & $793(41)$ & & $8.9 \%$ & & $13.1 \%$ & & 0.0023 \\
\hline ly1 & $1261(41)$ & $853(44)$ & & $14.9 \%$ & & $21.3 \%$ & & 0.0001 \\
\hline ly 2 & $378(12)$ & $262(13)$ & NS & $23.5 \%$ & $<0.0001$ & $32.4 \%$ & $<0.0001$ & 0.013 \\
\hline ly3 & $58(2)$ & $37(2)$ & & $48.3 \%$ & & $45.9 \%$ & & NS \\
\hline Unknown & $19(1)$ & $8(0)$ & & $10.5 \%$ & & $12.5 \%$ & & NS \\
\hline
\end{tabular}

(Continued) 


\begin{tabular}{|c|c|c|c|c|c|c|c|c|}
\hline \multirow{2}{*}{$\begin{array}{l}\text { Location } \\
\text { Factors }\end{array}$} & \multirow{2}{*}{$\begin{array}{c}\mathrm{CC} \\
(\mathrm{N}=3039) \\
\text { Number } \\
(\%)\end{array}$} & \multirow{2}{*}{$\begin{array}{c}\text { RC } \\
(\mathrm{N}=1953) \\
\text { Number } \\
(\%)\end{array}$} & \multirow{2}{*}{$\begin{array}{c}P \text { value } \\
\text { CC vs } \\
\text { RC }\end{array}$} & \multicolumn{2}{|c|}{$\mathrm{CC}$} & \multicolumn{2}{|c|}{$\mathbf{R C}$} & \multirow{2}{*}{$\begin{array}{c}P \text { value } \\
\text { CC vs } \\
\text { RC }\end{array}$} \\
\hline & & & & $\begin{array}{l}\text { Recurrence } \\
\text { rate }\end{array}$ & $P$ value & $\begin{array}{l}\text { Recurrence } \\
\text { rate }\end{array}$ & $P$ value & \\
\hline \multicolumn{9}{|l|}{ Venous invasion } \\
\hline v0 & $1193(39)$ & $610(31)$ & & $6.4 \%$ & & $10.5 \%$ & & 0.0025 \\
\hline v1 & $1188(39)$ & $765(39)$ & & $15.3 \%$ & & $19.3 \%$ & & 0.021 \\
\hline v2 & 493 (16) & $436(22)$ & $<0.0001$ & $23.5 \%$ & $<0.0001$ & $30.0 \%$ & $<0.0001$ & 0.025 \\
\hline v3 & $140(5)$ & $125(6)$ & & $32.1 \%$ & & $35.2 \%$ & & NS \\
\hline Unknown & $25(1)$ & $17(1)$ & & $20 \%$ & & $11.8 \%$ & & NS \\
\hline \multicolumn{9}{|l|}{ Stage } \\
\hline I & $890(29)$ & $641(33)$ & & $1.2 \%$ & & $8.4 \%$ & & $<0.0001$ \\
\hline II & $1149(38)$ & $614(31)$ & $<0.0001$ & $13.1 \%$ & $<0.0001$ & $20.0 \%$ & $<0.0001$ & 0.0002 \\
\hline III & $1000(33)$ & $697(36)$ & & $26.3 \%$ & & $30.4 \%$ & & NS \\
\hline \multicolumn{9}{|c|}{ Adjuvant therapy } \\
\hline \multicolumn{9}{|l|}{ Stage I } \\
\hline Yes & $18(2)$ & $26(4)$ & \multirow{2}{*}{0.019} & $5.6 \%$ & \multirow{2}{*}{ NS } & $7.7 \%$ & \multirow{2}{*}{ NS } & NS \\
\hline No & $872(98)$ & $615(96)$ & & $1.1 \%$ & & $8.5 \%$ & & $<0.0001$ \\
\hline \multicolumn{9}{|l|}{ Stage II } \\
\hline Yes & $184(16)$ & $126(20)$ & \multirow{2}{*}{0.019} & $17.9 \%$ & \multirow{2}{*}{0.036} & $25.4 \%$ & \multirow{2}{*}{ NS } & NS \\
\hline No & $965(84)$ & $489(80)$ & & $12.2 \%$ & & $18.6 \%$ & & 0.001 \\
\hline \multicolumn{9}{|l|}{ Stage III } \\
\hline Yes & $643(64)$ & $509(73)$ & \multirow{2}{*}{0.0002} & $25.8 \%$ & \multirow{2}{*}{ NS } & $31.0 \%$ & \multirow{2}{*}{ NS } & NS \\
\hline No & $357(36)$ & $188(27)$ & & $27.2 \%$ & & $28.7 \%$ & & NS \\
\hline
\end{tabular}

CEA: Carcinoembryonic antigen, CC: colon cancer, NS: Not significant, Poorly: poorly differentiated adenocarcinoma, Mucinous: mucinous adenocarcinoma.

RC: rectal cancer, Well/Moderately: well differentiated/moderately differentiated adenocarcinoma.

invasion, and distant lymph node metastasis (Table 3). TTR in stage III CC was significantly associated with preoperative CEA level, lymphatic invasion, and venous invasion (Table 3). Multivariate analysis showed that high preoperative CEA level and lymphatic invasion were independent predictors for short TTR in both stage II and stage III CC (Table 4).

TTR in stage II RC was significantly associated with metastatic factors (liver metastasis and distant lymph node metastasis), but not pathological factors. TTR in stage III RC was significantly associated with both pathological factors (lymphatic invasion and vascular invasion) and metastatic factors (M1b recurrence and liver metastasis) (Table 3). Multivariate analysis showed that venous invasion and liver metastasis were independent predictors for short TTR in stage III RC (Table 4).

\section{Survival after recurrence (SAR)}

The 5-year survival rates after recurrence for CC and $\mathrm{RC}$ were $81 \%$ and $77 \%$ in stage I; $55 \%$ and $58 \%$ in stage II; $40 \%$ and $39 \%$ in stage III, respectively (Figure $5 \mathrm{~F}-5 \mathrm{H})$. There were significant differences in the 5-year SAR rates among stages, but not between $\mathrm{CC}$ and $\mathrm{RC}$ (Figure 5F-5J).

SAR was significantly associated with age (all groups), gender (stage III RC), CEA level (stage I/II RC), histological type (stage III CRC), tumor depth (CC), nodal status (stage III CC), lymphatic invasion (stage III CRC), adjuvant therapy (stage III RC), recurrence within 1 year (stage III RC), M1b recurrence (stage II/III CRC), liver metastasis (stage II RC), peritoneal metastasis (CC and stage III RC), local recurrence (stage II CC), and treatment after recurrence (all groups) (Table 5). 

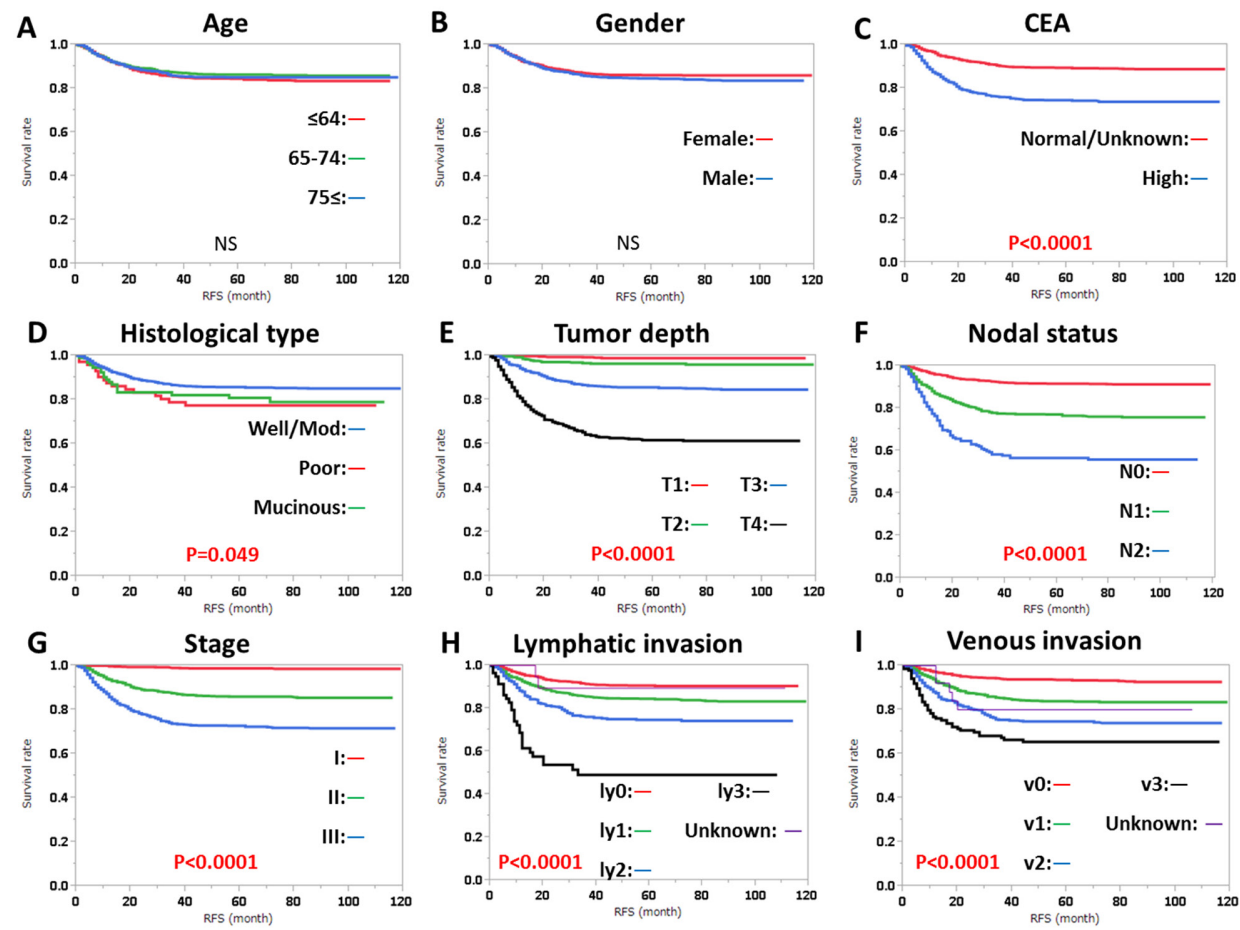

Figure 1: Relapse-free survival (RFS) in colon cancer according to (A) age, (B) gender, (C) CEA level, (D) histological type, (E) tumor depth, (F) nodal status, (G) clinical stage, (H) lymphatic invasion, and (I) venous invasion. Subgroups were compared with log-rank test. $\mathrm{P}$ values were provided when differences were significant $(\mathrm{P}<0.05)$ and as NS when differences were not significant.
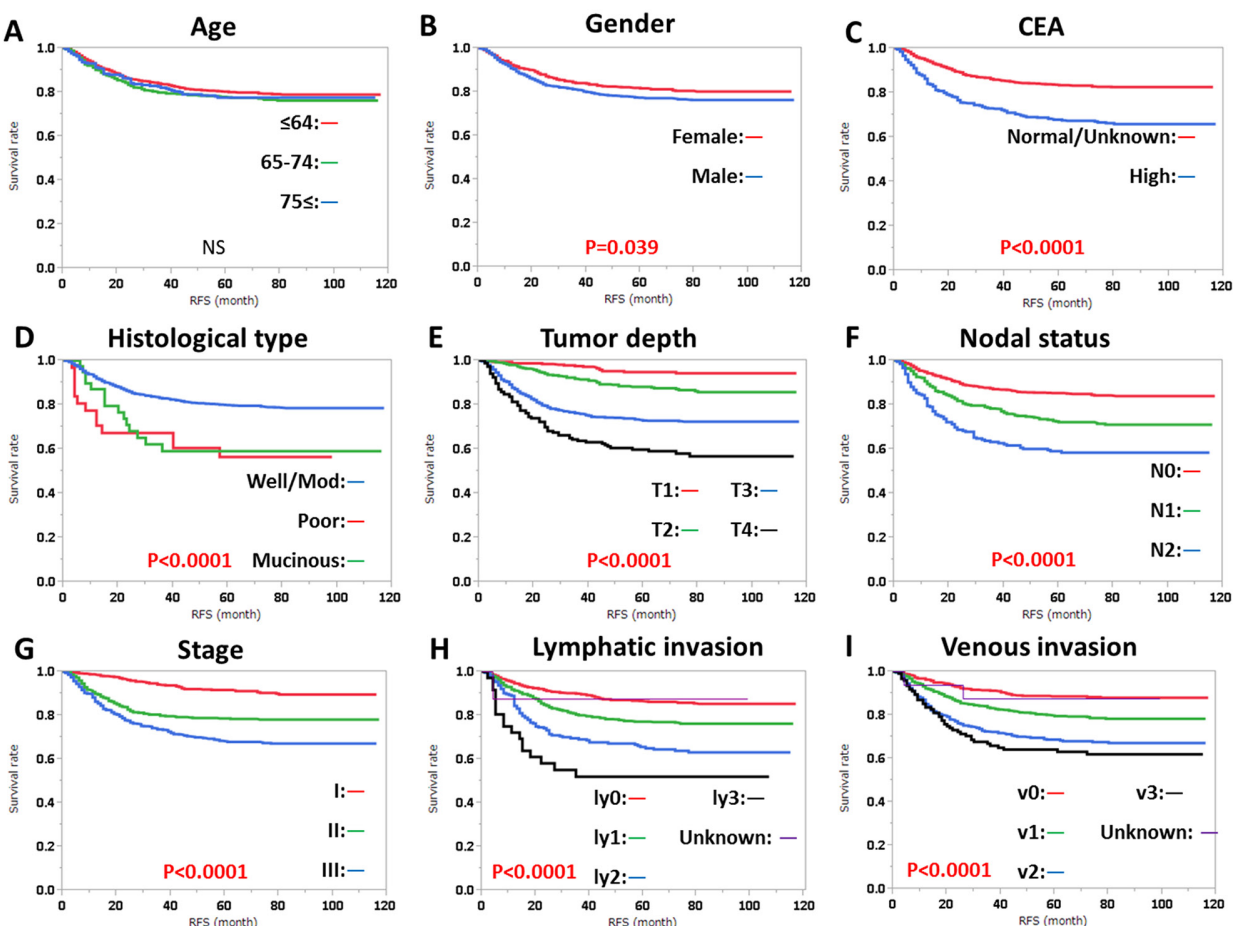

Figure 2: Relapse-free survival (RFS) in rectal cancer according to (A) age, (B) gender, (C) CEA level, (D) histological type, (E) tumor depth, (F) nodal status, (G) clinical stage, (H) lymphatic invasion, and (I) venous invasion. Subgroups were compared with log-rank test. $P$ values were provided when differences were significant $(\mathrm{P}<0.05)$ and as NS when differences were not significant. 

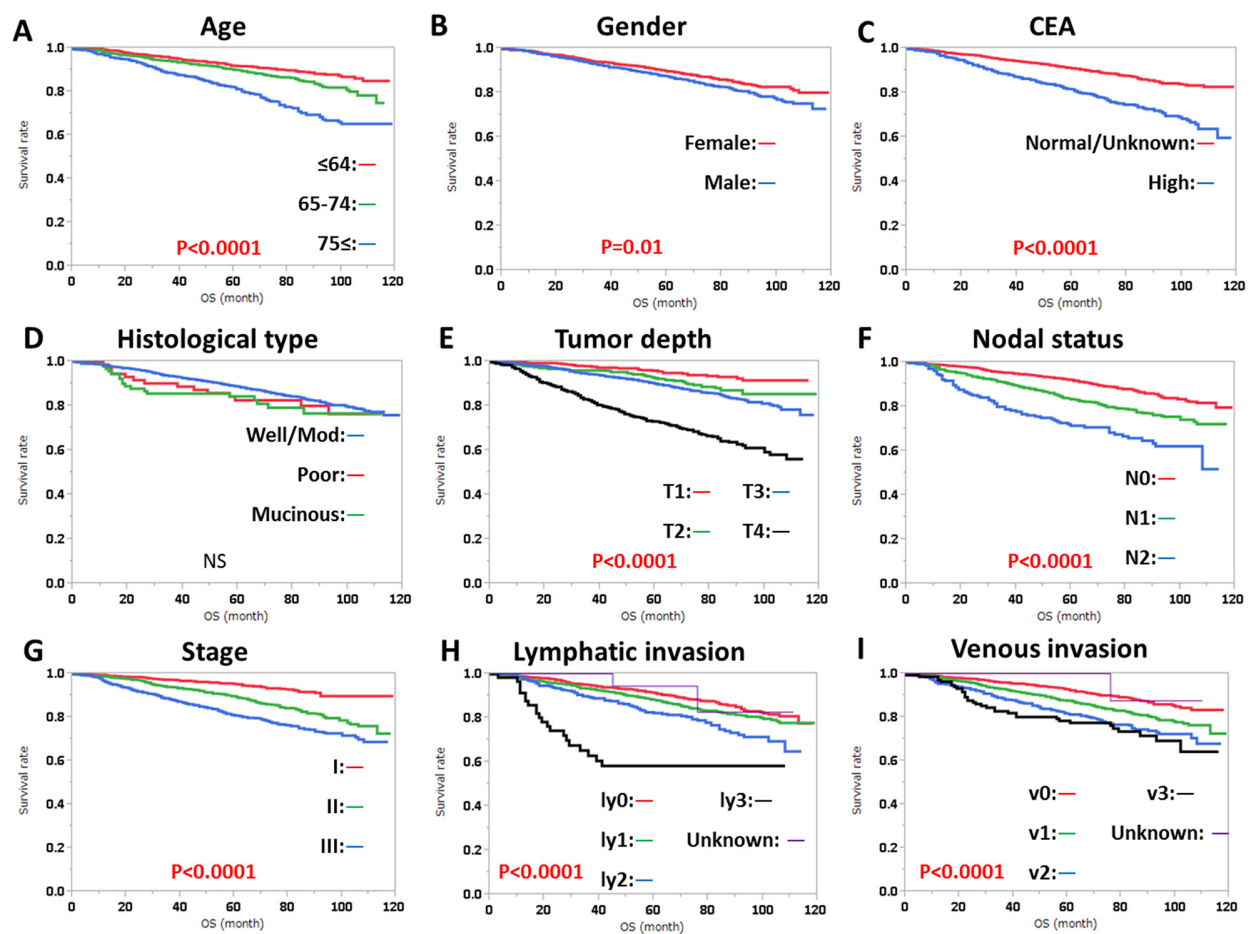

Figure 3: Overall survival (OS) in colon cancer according to (A) age, (B) gender, (C) CEA level, (D) histological type, (E) tumor depth, (F) nodal status, (G) clinical stage, (H) lymphatic invasion, and (I) venous invasion. Subgroups were compared with log-rank test. P values were provided when differences were significant $(\mathrm{P}<0.05)$ and as NS when differences were not significant.
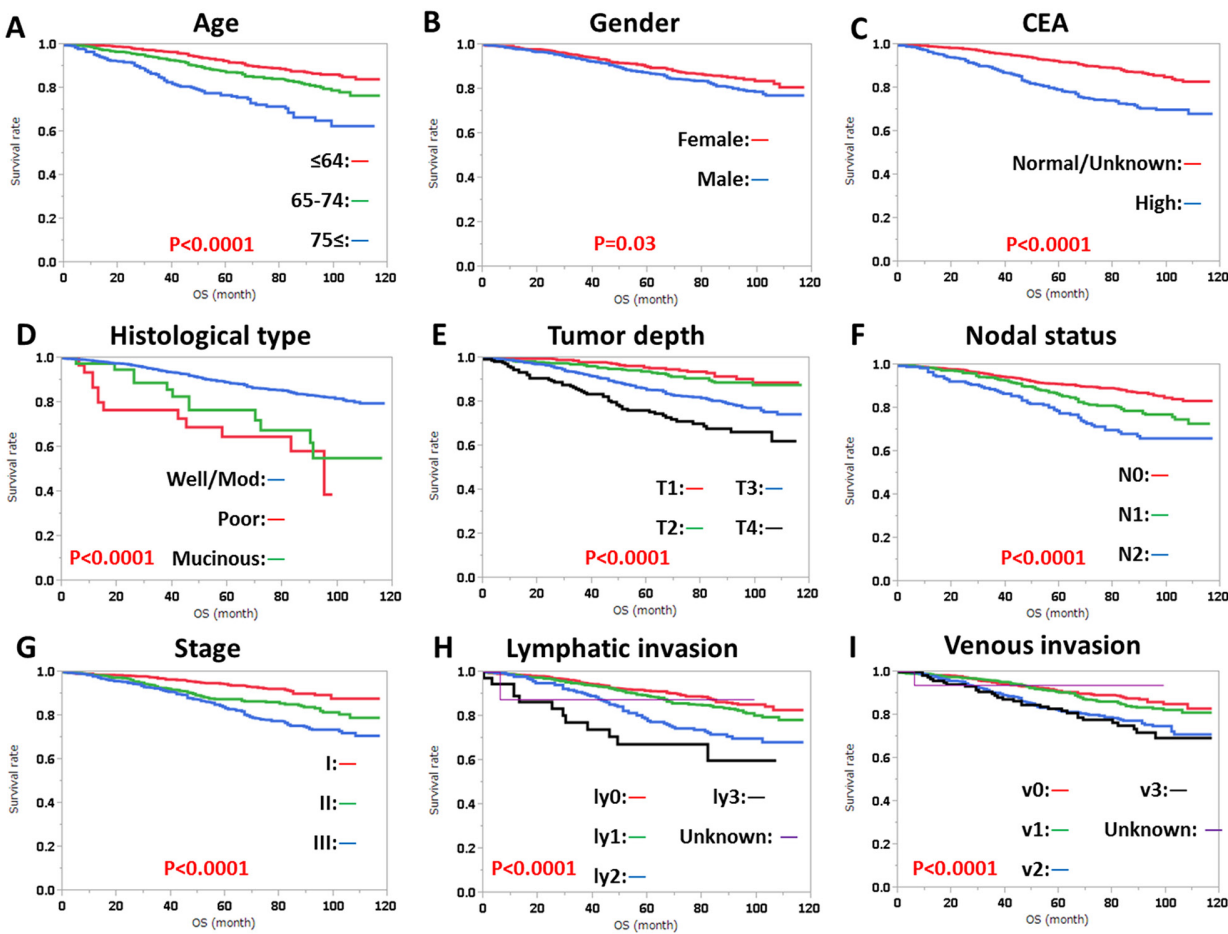

Figure 4: Overall survival (OS) in rectal cancer according to (A) age, (B) gender, (C) CEA level, (D) histological type, (E) tumor depth, (F) nodal status, (G) clinical stage, (H) lymphatic invasion, and (I) venous invasion. Subgroups were compared with log-rank test. P values were provided when differences were significant $(\mathrm{P}<0.05)$. 
Table 2: Clinicopathological factors of patients with recurrence by tumor location

\begin{tabular}{|c|c|c|c|}
\hline Factors / Location & $\begin{array}{l}\mathrm{CC}(\mathrm{N}=425) \\
\text { Number }(\%)\end{array}$ & $\begin{array}{l}\mathrm{RC}(\mathrm{N}=389) \\
\text { Number }(\%)\end{array}$ & $P$ value $C C$ vs $R C$ \\
\hline \multicolumn{4}{|l|}{ Age } \\
\hline$\leq 64 / 65-74 / 75 \leq$ & $\begin{array}{c}160 / 143 / 122 \\
(38 / 33 / 29)\end{array}$ & $\begin{array}{l}193 / 136 / 60 \\
(50 / 35 / 15)\end{array}$ & $<0.0001$ \\
\hline \multicolumn{4}{|l|}{ Gender } \\
\hline Male / Female & $\begin{array}{c}248 / 177 \\
(58 / 42)\end{array}$ & $\begin{array}{c}255 / 134 \\
(66 / 34)\end{array}$ & 0.035 \\
\hline \multicolumn{4}{|l|}{ Preoperative CEA } \\
\hline $\begin{array}{l}\text { High / Normal / } \\
\text { Unknown }\end{array}$ & $\begin{array}{l}199 / 217 / 9 \\
(47 / 51 / 2)\end{array}$ & $\begin{array}{c}171 / 201 / 17 \\
(44 / 52 / 4)\end{array}$ & NS \\
\hline \multicolumn{4}{|l|}{ Histological Type } \\
\hline $\begin{array}{l}\text { Well/Moderately / } \\
\text { Poorly /Mucinous }\end{array}$ & $\begin{array}{c}391 / 16 / 18 \\
(92 / 2 / 2)\end{array}$ & $\begin{array}{c}361 / 13 / 15 \\
(93 / 3 / 4)\end{array}$ & NS \\
\hline \multicolumn{4}{|l|}{ Tumor depth } \\
\hline $\mathrm{T} 1 / \mathrm{T} 2$ / T3 / T4 & $\begin{array}{c}6 / 16 / 197 / 206 \\
(1 / 4 / 46 / 48)\end{array}$ & $\begin{array}{l}17 / 52 / 247 / 73 \\
(4 / 13 / 63 / 19)\end{array}$ & $<0.0001$ \\
\hline \multicolumn{4}{|l|}{ Nodal status } \\
\hline $\mathrm{N} 0 / \mathrm{N} 1 / \mathrm{N} 2$ & $\begin{array}{l}162 / 175 / 88 \\
(38 / 41 / 21)\end{array}$ & $\begin{array}{l}177 / 127 / 85 \\
(46 / 33 / 22)\end{array}$ & 0.034 \\
\hline \multicolumn{4}{|l|}{ Lymphatic invasion } \\
\hline $\begin{array}{l}\operatorname{ly} 0 \text { / ly1 / ly2 / ly3/ } \\
\text { Unknown }\end{array}$ & $\begin{array}{c}118 / 188 / 89 / 28 / 2 \\
(28 / 44 / 21 / 7 / 1)\end{array}$ & $\begin{array}{c}104 / 182 / 85 / 17 / 1 \\
(27 / 47 / 22 / 4 / 0)\end{array}$ & NS \\
\hline \multicolumn{4}{|l|}{ Venous invasion } \\
\hline $\begin{array}{l}\text { v0 / v1 / v2 / v3 / } \\
\text { Unknown }\end{array}$ & $\begin{array}{c}77 / 182 / 116 / 45 / 5 \\
(18 / 43 / 27 / 11 / 1)\end{array}$ & $\begin{array}{c}64 / 148 / 131 / 44 / 2 \\
(16 / 38 / 34 / 11 / 1)\end{array}$ & NS \\
\hline \multicolumn{4}{|l|}{ Stage } \\
\hline I / II / III & $\begin{array}{c}11 / 151 / 263 \\
(3 / 36 / 62)\end{array}$ & $\begin{array}{l}54 / 123 / 212 \\
(14 / 32 / 54)\end{array}$ & $<0.0001$ \\
\hline \multicolumn{4}{|l|}{ Time to recurrence (year) } \\
\hline$<1 / 1-2 / 2-3 / 3 \leq$ & $\begin{array}{c}190 / 136 / 64 / 35 \\
(45 / 32 / 15 / 8)\end{array}$ & $\begin{array}{l}142 / 122 / 57 / 68 \\
(37 / 31 / 15 / 17)\end{array}$ & 0.0007 \\
\hline \multicolumn{4}{|l|}{ Type of recurrence } \\
\hline Local alone /M1a / M1b & $\begin{array}{c}29 / 251 / 145 \\
(7 / 59 / 34)\end{array}$ & $\begin{array}{l}77 / 243 / 69 \\
(20 / 62 / 18)\end{array}$ & $<0.0001$ \\
\hline \multicolumn{4}{|l|}{ Liver metastasis } \\
\hline$(+) /(-)$ & $\begin{array}{c}203 / 222 \\
(48 / 52)\end{array}$ & $\begin{array}{l}128 / 261 \\
(33 / 67)\end{array}$ & $<0.0001$ \\
\hline \multicolumn{4}{|l|}{ Lung metastasis } \\
\hline$(+) /(-)$ & $\begin{array}{l}130 / 295 \\
(31 / 69)\end{array}$ & $\begin{array}{l}148 / 241 \\
(38 / 62)\end{array}$ & 0.025 \\
\hline
\end{tabular}

(Continued) 


\begin{tabular}{lccc}
\hline Factors / Location & $\begin{array}{c}\text { CC }(\mathbf{N}=\mathbf{4 2 5}) \\
\text { Number (\%) }\end{array}$ & $\begin{array}{c}\text { RC }(\mathbf{N}=\mathbf{3 8 9}) \\
\text { Number (\%) }\end{array}$ & P value CC vs RC \\
\hline Peritoneal metastasis & & & \\
$(+) /(-)$ & $(20 / 80)$ & $28 / 361$ & $<0.0001$ \\
Local metastasis & & $(7 / 93)$ & \\
$(+) /(-)$ & $(10 / 90)$ & $112 / 277$ & $<0.0001$ \\
Distant lymph node metastasis & & $(29 / 71)$ & \\
$(+) /(-)$ & $63 / 362$ & & NS \\
Adjuvant therapy & $(15 / 85)$ & $(12 / 88)$ & \\
$(+) /(-)$ & & & NS \\
Treatment after recurrence & $200 / 225$ & $192 / 197$ & \\
Best supportive care / & $(47 / 53)$ & $(49 / 51)$ & NS \\
Surgery (-) / Surgery $(+)$ & $38 / 188 / 199$ & & \\
\hline
\end{tabular}

CEA: Carcinoembryonic antigen, CC: colon cancer, Mucinous: mucinous adenocarcinoma, NS: Not significant, Poorly: poorly differentiated adenocarcinoma, RC: rectal cancer, Well/Moderately: well differentiated/moderately differentiated adenocarcinoma
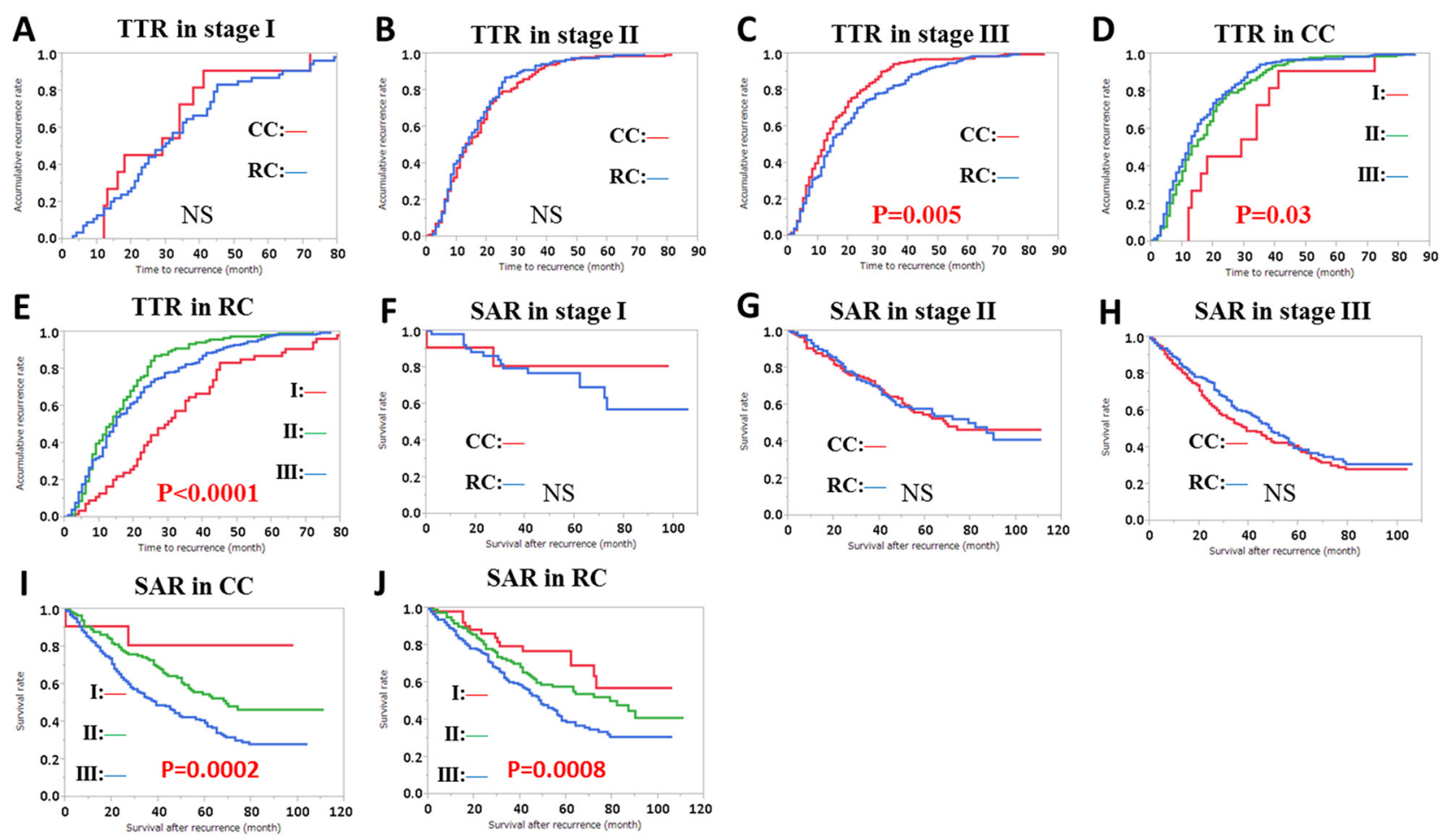

Figure 5: Time to recurrence (TTR) and accumulative recurrence rate (A-E), and survival after recurrence (SAR) and survival rate (F-J), by clinicopathological factors. TTR and accumulative recurrence rate in: (A) stage I patients by colon cancer (CC) and rectal cancer (RC), (B) stage II patients by CC and RC, (C) stage III patients by CC and RC, (D) CC by stage, (E) RC by stage. SAR and survival rate in: (F) stage I patients by CC and RC, (G) stage II patients by CC and RC, (H) stage III patients by CC and RC, (I) CC by stage, (J) RC by stage. Subgroups were compared with log-rank test. $\mathrm{P}$ values were provided when differences were significant $(\mathrm{P}<0.05)$ and NS was used when differences were not significant. 
Table 3: Summary of associations between clinicopathological factors and TTR

\begin{tabular}{|c|c|c|c|c|c|c|c|c|}
\hline \multirow{3}{*}{$\begin{array}{l}\text { Location } \\
\text { Stage } \\
\text { Factors }\end{array}$} & \multicolumn{4}{|c|}{ Colon cancer } & \multicolumn{4}{|c|}{ Rectal cancer } \\
\hline & \multicolumn{2}{|c|}{ Stage II (N=151) } & \multicolumn{2}{|c|}{ Stage III $(N=263)$} & \multicolumn{2}{|c|}{ Stage II $(\mathrm{N}=123)$} & \multicolumn{2}{|c|}{ Stage III $(\mathrm{N}=\mathbf{2 1 2})$} \\
\hline & $\begin{array}{c}\text { Mean } \\
\text { (months) }\end{array}$ & $P$ & $\begin{array}{c}\text { Mean } \\
\text { (months) }\end{array}$ & $P$ & $\begin{array}{c}\text { Mean } \\
\text { (months) }\end{array}$ & $P$ & $\begin{array}{c}\text { Mean } \\
\text { (months) }\end{array}$ & $P$ \\
\hline \multicolumn{9}{|l|}{ Age } \\
\hline$\leq 74$ & 17.5 & \multirow{2}{*}{ NS } & 16.6 & \multirow{2}{*}{ NS } & 16.2 & \multirow{2}{*}{ NS } & 20.0 & \multirow{2}{*}{ NS } \\
\hline $75 \leq$ & 18.0 & & 13.9 & & 17.4 & & 19 & \\
\hline \multicolumn{9}{|l|}{ Gender } \\
\hline Male & 19.6 & \multirow{2}{*}{0.04} & 16.5 & \multirow{2}{*}{ NS } & 16.9 & \multirow{2}{*}{ NS } & 20.5 & \multirow{2}{*}{ NS } \\
\hline Female & 15.3 & & 14.8 & & 15.3 & & 18.7 & \\
\hline \multicolumn{9}{|l|}{ Preoperative CEA } \\
\hline High & 14.7 & \multirow{2}{*}{0.02} & 14.1 & \multirow{2}{*}{0.04} & 16 & \multirow{2}{*}{ NS } & 18.8 & \multirow{2}{*}{ NS } \\
\hline Normal/Unknown & 20.3 & & 17.5 & & 16.7 & & 20.9 & \\
\hline \multicolumn{9}{|l|}{ Histological Type } \\
\hline Well/Moderately & 17.5 & \multirow{2}{*}{ NS } & 16.0 & \multirow{2}{*}{ NS } & 16.3 & \multirow{2}{*}{ NS } & 20.2 & NS \\
\hline Poorly/Mucinous & 20.5 & & 14.1 & & 18 & & 16.7 & S \\
\hline Tumor depth & & & & & & & & \\
\hline $\mathrm{T} 1$ & - & & 15.3 & & & & 28.5 & \\
\hline $\mathrm{T} 2$ & - & NS & 14.9 & NS & & NS & 30.7 & NS \\
\hline $\mathrm{T} 3$ & 19.3 & IVI & 13.9 & 1VI & 15.7 & נIV & 19.2 & N \\
\hline $\mathrm{T} 4$ & 15.6 & & 15.8 & & 19.2 & & 18.7 & \\
\hline Nodal status & & & & & & & & \\
\hline No & & & & & & & & \\
\hline N1 & 17.7 & - & 16.3 & NS & 16.4 & - & 21.8 & 0.03 \\
\hline $\mathrm{N} 2$ & & & 14.9 & & & & 16.9 & \\
\hline Lymphatic invasion & & & & & & & & \\
\hline ly $0 / 1$ & 18.5 & 0008 & 16.7 & NS & 15.8 & NS & 16.7 & NS \\
\hline ly $2 / 3$ & 11.4 & 0.000 & 15.1 & NDS & 20.4 & NS & 22.0 & NDS \\
\hline ly0-2 & 17.8 & NS & 16.4 & 0008 & 16.4 & & 20.6 & 0000 \\
\hline ly3 & 13 & Nis & 10.3 & 0.000 & - & - & 11.9 & 0.009 \\
\hline Venous invasion & & & & & & & & \\
\hline $\mathrm{v} 0 / 1$ & 18.7 & & 17.2 & & 15.3 & & 22.4 & \\
\hline $\mathrm{v} 2 / 3$ & 15.7 & & 14.0 & 10. & 17.5 & & 17.1 & \\
\hline $\mathrm{v} 0-2$ & 17.9 & NS & 16.6 & 000 & 16.3 & XIS & 20.0 & DT \\
\hline v3 & 15.2 & NS & 9.7 & 0.001 & 16.9 & NS & 18.9 & NS \\
\hline Adjuvant therapy & & & & & & & & \\
\hline Yes & 16.3 & NC & 16.9 & NS & 13.9 & NS & 19.7 & NS \\
\hline No & 18.1 & IVI & 14.0 & 1Vis & 17.3 & Div & 20.3 & IV \\
\hline
\end{tabular}




\begin{tabular}{|c|c|c|c|c|c|c|c|c|}
\hline \multirow{3}{*}{$\begin{array}{l}\text { Location } \\
\text { Stage } \\
\text { Factors }\end{array}$} & \multicolumn{4}{|c|}{ Colon cancer } & \multicolumn{4}{|c|}{ Rectal cancer } \\
\hline & \multicolumn{2}{|c|}{ Stage II $(\mathrm{N}=151)$} & \multicolumn{2}{|c|}{ Stage III (N=263) } & \multicolumn{2}{|c|}{ Stage II $(N=123)$} & \multicolumn{2}{|c|}{ Stage III $(\mathrm{N}=212)$} \\
\hline & $\begin{array}{c}\text { Mean } \\
\text { (months) }\end{array}$ & $\boldsymbol{P}$ & $\begin{array}{c}\text { Mean } \\
\text { (months) }\end{array}$ & $P$ & $\begin{array}{c}\text { Mean } \\
\text { (months) }\end{array}$ & $P$ & $\begin{array}{c}\text { Mean } \\
\text { (months) }\end{array}$ & $P$ \\
\hline \multicolumn{9}{|c|}{ Type of recurrence } \\
\hline M1a & 18.8 & \multirow{3}{*}{ NS } & 15.5 & \multirow{3}{*}{ NS } & 17.4 & \multirow{3}{*}{ NS } & 21.0 & \multirow{3}{*}{0.02} \\
\hline M1b & 15.0 & & 15.2 & & 13 & & 14.8 & \\
\hline Local alone & 20.5 & & 23.4 & & 15.7 & & 22.9 & \\
\hline \multicolumn{9}{|l|}{ Liver metastasis } \\
\hline$(-)$ & 18.6 & \multirow{2}{*}{ NS } & 16.9 & \multirow{2}{*}{ NS } & 18.6 & \multirow{2}{*}{0.01} & 23.0 & \multirow{2}{*}{$<0.0001$} \\
\hline$(+)$ & 16.8 & & 14.5 & & 13 & & 12.8 & \\
\hline \multicolumn{9}{|l|}{ Lung metastasis } \\
\hline$(-)$ & 17.2 & \multirow{2}{*}{ NS } & 15.6 & \multirow{2}{*}{ NS } & 15.8 & \multirow{2}{*}{ NS } & 19.4 & \multirow{2}{*}{ NS } \\
\hline$(+)$ & 18.5 & & 16.2 & & 17.6 & & 20.4 & \\
\hline \multicolumn{9}{|c|}{ Peritoneal metastasis } \\
\hline$(-)$ & 17.8 & \multirow{2}{*}{ NS } & 16.2 & \multirow{2}{*}{ NS } & 16.4 & \multirow{2}{*}{ NS } & 20.2 & \multirow{2}{*}{ NS } \\
\hline$(+)$ & 16.9 & & 14.3 & & 16 & & 17.3 & \\
\hline \multicolumn{9}{|c|}{ Local recurrence } \\
\hline$(-)$ & 17.6 & \multirow{2}{*}{ NS } & 15.5 & \multirow{2}{*}{ NS } & 16.8 & \multirow{2}{*}{ NS } & 19.4 & \multirow{2}{*}{ NS } \\
\hline$(+)$ & 18.1 & & 18.9 & & 15.4 & & 21.1 & \\
\hline \multicolumn{9}{|c|}{ Distant LN metastasis } \\
\hline$(-)$ & 18.3 & \multirow{2}{*}{0.04} & 15.2 & & 27.7 & & 19.8 & \\
\hline$(+)$ & 11.9 & & 18.4 & NOS & 15.5 & 0.02 & 20.4 & NOS \\
\hline
\end{tabular}

CEA: Carcinoembryonic antigen, LN: lymph node, Mucinous: mucinous adenocarcinoma, NS: Not significant, Poorly: poorly differentiated adenocarcinoma, TTR: time to recurrence, Well/Moderately: well differentiated/moderately differentiated adenocarcinoma.

Multivariate analysis identified the following independent prognostic factors: age (stage II CC and stage III RC), female gender (stage III RC), high CEA level (stage II RC), histological type (stage III CRC), nodal status (stage III CC), recurrence within 1 year (stage III $\mathrm{RC}$ ), M1b recurrence (stage II CRC), local recurrence (stage II CC), and no surgical resection after recurrence (stage II and III CRC) (Table 6).

\section{DISCUSSION}

We evaluated the clinicopathological factors associated with TTR and SAR in patients with $\mathrm{CC}$ and $\mathrm{RC}$, as critical factors for guiding appropriate followup after curative surgery [3-5]. Appropriate follow-up after curative surgery has been proposed by TTR or the accumulative recurrence rate, but not SAR.

The recurrence rate in this study $(16.3 \%)$ was similar to those in previous studies $[3,7]$. The recurrence rate in stage I CC patients was only $1.2 \%$, so we excluded these patients from analysis of TTR and SAR. The recurrence rate in stage I RC patients was $8.4 \%$, and the accumulative recurrence rates were $16.7 \%$ and $64.8 \%$ at 1 and 3 years, respectively. Therefore, stage I RC patients may require different surveillance from stage II and III patients. We also excluded stage I RC patients for further analysis of TTR and SAR.

Among stage II and III patients, the accumulative recurrence rate was about $50 \%$ at 1 year, which was higher than in some previous reports, but similar to that in the FACS trial involving intensive surveillance, including CT $[3,7]$. The 2- and 3-year recurrence rates in patients with stages II and III were $70 \%-80 \%$ and $80 \%-90 \%$, respectively. These are similar to the previous data by Kobayashi et al and suggest that intensive surveillance with CEA checks every 3 months are necessary for at least 3 years, in contrast to the NCCN recommendation of 2 years $[3,14,15]$. 
Table 4: Multivariate analysis of predictors for short TTR

\begin{tabular}{|c|c|c|c|c|c|c|c|c|}
\hline \multirow{3}{*}{$\begin{array}{l}\text { Location } \\
\text { Stage } \\
\text { Factors }\end{array}$} & \multicolumn{4}{|c|}{ Colon cancer } & \multicolumn{4}{|c|}{ Rectal cancer } \\
\hline & \multicolumn{2}{|c|}{ Stage II } & \multicolumn{2}{|c|}{ Stage III } & \multicolumn{2}{|c|}{ Stage II } & \multicolumn{2}{|c|}{ Stage III } \\
\hline & $\begin{array}{c}\text { HR } \\
\text { CI }\end{array}$ & $P$ & $\begin{array}{c}\text { HR } \\
\text { CI }\end{array}$ & $\boldsymbol{P}$ & $\begin{array}{c}\text { HR } \\
\text { CI }\end{array}$ & $P$ & $\begin{array}{c}\text { HR } \\
\text { CI }\end{array}$ & $P$ \\
\hline Gender: Male & $\begin{array}{c}0.73 \\
0.53- \\
1.02\end{array}$ & NS & & & & & & \\
\hline CEA: High & $\begin{array}{l}1.47 \\
1.06- \\
2.03\end{array}$ & 0.02 & $\begin{array}{c}1.34 \\
1.04- \\
1.73\end{array}$ & 0.02 & & & & \\
\hline Nodal status: N2 & & & & & & & $\begin{array}{c}1.23 \\
0.92- \\
1.64\end{array}$ & NS \\
\hline \multicolumn{9}{|l|}{ Lymphatic invasion } \\
\hline $\operatorname{ly} 2 / 3$ & $\begin{array}{c}1.83 \\
1.03- \\
3.05\end{array}$ & 0.04 & & & & & & \\
\hline $\operatorname{ly} 3$ & & & $\begin{array}{l}1.73 \\
1.09- \\
2.64\end{array}$ & 0.02 & & & $\begin{array}{c}1.5 \\
0.86- \\
2.45\end{array}$ & NS \\
\hline \multicolumn{9}{|l|}{ Venous invasion } \\
\hline $\mathrm{v} 2 / 3$ & & & & & & & $\begin{array}{c}1.42 \\
1.07- \\
1.88\end{array}$ & 0.01 \\
\hline $\mathrm{v} 3$ & & & $\begin{array}{c}1.63 \\
1.09- \\
2.36\end{array}$ & 0.02 & & & & \\
\hline \multicolumn{9}{|l|}{ Type of recurrence: } \\
\hline M1b & & & & & & & $\begin{array}{c}1.25 \\
0.88- \\
1.74\end{array}$ & NS \\
\hline \multicolumn{9}{|l|}{ Recurrence sites } \\
\hline Liver $(+)$ & & & & & $\begin{array}{c}1.44 \\
0.99- \\
2.09\end{array}$ & NS & $\begin{array}{c}1.92 \\
1.41- \\
2.61\end{array}$ & $<0.0001$ \\
\hline Distant LN: $(+)$ & $\begin{array}{c}1.53 \\
0.84- \\
2.61\end{array}$ & NS & & & $\begin{array}{c}0.52 \\
0.23- \\
1.03\end{array}$ & NS & & \\
\hline
\end{tabular}

CEA: Carcinoembryonic antigen, CI: confidence interval, HR: hazard ratio, LN: lymph node, NS: Not significant, TTR: time to recurrence.

TTR differed significantly between $\mathrm{CC}$ and RC in stage III patients. Predictors for TTR were different between $\mathrm{CC}$ and $\mathrm{RC}$. In $\mathrm{CC}$, patients with high preoperative CEA level and lymphovascular invasion could be followed-up as candidates for early recurrence. On the other hand, predictors in $\mathrm{RC}$ were venous invasion and liver metastasis. Therefore, follow-up in RC should include CT to evaluate liver metastasis.

Our data showed that predictors for TTR were not prognostic factors for SAR. Short TTR has been previously reported to influence survival in small cases of study $[16,17]$. In this study, recurrence within 1 year 
Table 5: Summary of associations between clinicopathological factors and SAR

\begin{tabular}{|c|c|c|c|c|c|c|c|c|}
\hline \multirow{3}{*}{$\begin{array}{l}\text { Location } \\
\text { Stage } \\
\text { Factors }\end{array}$} & \multicolumn{4}{|c|}{ Colon cancer } & \multicolumn{4}{|c|}{ Rectal cancer } \\
\hline & \multicolumn{2}{|c|}{ Stage II (N=151) } & \multicolumn{2}{|c|}{ Stage III $(N=263)$} & \multicolumn{2}{|c|}{ Stage II $(\mathrm{N}=123)$} & \multicolumn{2}{|c|}{ Stage III $(\mathrm{N}=\mathbf{2 1 2})$} \\
\hline & $\begin{array}{c}\text { Mean } \\
\text { (months) }\end{array}$ & $P$ & Mean (months) & $P$ & $\begin{array}{c}\text { Mean } \\
\text { (months) }\end{array}$ & $P$ & $\begin{array}{c}\text { Mean } \\
\text { (months) }\end{array}$ & $P$ \\
\hline \multicolumn{9}{|l|}{ Age } \\
\hline$\leq 74$ & 59.1 & \multirow{2}{*}{0.0001} & 46.9 & \multirow{2}{*}{0.005} & 64.2 & \multirow{2}{*}{0.01} & 51.1 & \multirow{2}{*}{$<0.0001$} \\
\hline $75 \leq$ & 33.3 & & 34.1 & & 35.0 & & 24.2 & \\
\hline \multicolumn{9}{|l|}{ Gender } \\
\hline Male & 51.9 & \multirow{2}{*}{ NS } & 44.9 & \multirow{2}{*}{ NS } & 59.5 & \multirow{2}{*}{ NS } & 51.6 & \multirow{2}{*}{0.004} \\
\hline Female & 51.9 & & 41.5 & & 64.7 & & 39.6 & \\
\hline \multicolumn{9}{|l|}{ Preoperative CEA } \\
\hline High & 48.8 & \multirow{2}{*}{ NS } & 44.9 & \multirow{2}{*}{ NS } & 46.8 & \multirow{2}{*}{0.049} & 45.6 & \multirow{2}{*}{ NS } \\
\hline Normal/Unknown & 55.4 & & 41.6 & & 67.9 & & 50.0 & \\
\hline \multicolumn{9}{|l|}{ Histological Type } \\
\hline Well/Moderately & 52.6 & \multirow{2}{*}{ NS } & 45.8 & \multirow{2}{*}{0.003} & 61.8 & \multirow{2}{*}{ NS } & 50.3 & \multirow{2}{*}{$<0.0001$} \\
\hline Poorly/Mucinous & 44.5 & & 18.4 & & 17.4 & & 23.8 & \\
\hline Tumor depth & & & & & & & & \\
\hline $\mathrm{T} 1$ & & & 16 & & & & 55 & \\
\hline $\mathrm{T} 2$ & & 0000 & 34.7 & ר000 & & NS & 42.8 & NS \\
\hline $\mathrm{T} 3$ & 49.3 & 0.00 & 53.4 & 0.002 & 62.8 & תוני & 49.7 & N \\
\hline $\mathrm{T} 4$ & 48.4 & & 35.8 & & 55.9 & & 48.0 & \\
\hline Nodal status & & & & & & & & \\
\hline N1 & & & 47.1 & $00 \Omega$ & & & 50.3 & NS \\
\hline N2 & & & 36.4 & 0.02 & & & 41.5 & \\
\hline Lymphatic invasion & & & & & & & & \\
\hline $\operatorname{ly} 0 / 1$ & 51.4 & & 46.5 & & 63.2 & IS & 50.7 & \\
\hline $\operatorname{ly} 2 / 3$ & 49.6 & IVS & 38.7 & INS & 37.2 & IV & 40.7 & IV. \\
\hline ly0-2 & 53.4 & NS & 45.5 & 0004 & 61.6 & & 49.2 & 0005 \\
\hline ly3 & 14.7 & 10. & 16.3 & 0.004 & - & & 29.6 & $0.00 J$ \\
\hline Venous invasion & & & & & & & & \\
\hline $\mathrm{v} 0 / 1$ & 51.5 & NS & 44.0 & NS & 65.8 & NS & 48.9 & NS \\
\hline$v 2 / 3$ & 51.3 & 10. & 43.3 & מט & 54.3 & 10. & 45.6 & 10. \\
\hline v0-2 & 50.6 & NS & 44.3 & NS & 60.7 & NS & 48.3 & NS \\
\hline v3 & 58.1 & $1 \mathrm{~N}$ & 38.8 & IV.S & 53.7 & IV & 44.2 & Niv \\
\hline Adjuvant therapy & & & & & & & & \\
\hline Yes & 57.0 & & 45.0 & & 65.5 & & 50.9 & \\
\hline No & 50.9 & 1V. & 41.1 & מת & 58.8 & 1 & 37.7 & 0.02 \\
\hline
\end{tabular}




\begin{tabular}{|c|c|c|c|c|c|c|c|c|}
\hline \multirow{3}{*}{$\begin{array}{l}\text { Location } \\
\text { Stage } \\
\text { Factors }\end{array}$} & \multicolumn{4}{|c|}{ Colon cancer } & \multicolumn{4}{|c|}{ Rectal cancer } \\
\hline & \multicolumn{2}{|c|}{ Stage II (N=151) } & \multicolumn{2}{|c|}{ Stage III $(N=263)$} & \multicolumn{2}{|c|}{ Stage II (N=123) } & \multicolumn{2}{|c|}{ Stage III $(\mathrm{N}=212)$} \\
\hline & $\begin{array}{c}\text { Mean } \\
\text { (months) }\end{array}$ & $P$ & Mean (months) & $P$ & $\begin{array}{c}\text { Mean } \\
\text { (months) }\end{array}$ & $P$ & $\begin{array}{c}\text { Mean } \\
\text { (months) }\end{array}$ & $P$ \\
\hline \multicolumn{9}{|l|}{ Time to recurrence } \\
\hline less than 1 year & 48.9 & \multirow{2}{*}{ NS } & 37.8 & \multirow{2}{*}{ NS } & 58.1 & \multirow{2}{*}{ NS } & 41.2 & \multirow{2}{*}{0.004} \\
\hline more than 1 year & 53.0 & & 48.1 & & 62.9 & & 50.4 & \\
\hline \multicolumn{9}{|l|}{ Type of recurrence } \\
\hline M1a & 59.0 & \multirow{3}{*}{0.0002} & 49.8 & \multirow{3}{*}{0.0001} & 61.2 & \multirow{3}{*}{0.001} & 53.6 & \multirow{3}{*}{0.0002} \\
\hline M1b & 43.8 & & 33.6 & & 35.2 & & 29.6 & \\
\hline Local alone & 42.7 & & 46.8 & & 64.9 & & 43.2 & \\
\hline \multicolumn{9}{|l|}{ Liver metastasis } \\
\hline$(-)$ & 46.7 & \multirow{2}{*}{0.01} & 41.3 & \multirow{2}{*}{ NS } & 60.4 & \multirow{2}{*}{ NS } & 47.4 & \multirow{2}{*}{ NS } \\
\hline$(+)$ & 57.8 & & 46.3 & & 48.9 & & 48.4 & \\
\hline \multicolumn{9}{|l|}{ Lung metastasis } \\
\hline$(-)$ & 56.0 & \multirow{2}{*}{ NS } & 42.8 & \multirow{2}{*}{ NS } & 65.9 & \multirow{2}{*}{0.02} & 46.0 & \multirow{2}{*}{ NS } \\
\hline$(+)$ & 42.7 & & 45.0 & & 46.3 & & 49.0 & \\
\hline \multicolumn{9}{|l|}{ Peritoneal metastasis } \\
\hline$(-)$ & 54.2 & \multirow{2}{*}{0.03} & 47.8 & \multirow{2}{*}{$<0.0001$} & 62.5 & & 50.0 & 0001 \\
\hline$(+)$ & 45.2 & & 29.3 & & 15.4 & NS & 21.8 & 0.001 \\
\hline Local recurrence & & & & & & & & \\
\hline$(-)$ & 54.1 & & 44.1 & & 59.6 & & 48.3 & \\
\hline$(+)$ & 42.6 & 0.04 & 41.2 & NiS & 57.0 & NiS & 46.0 & No \\
\hline Distant LN metastasi & & & & & & & & \\
\hline$(-)$ & 53.7 & NS & 44.5 & NS & 61.2 & NS & 49.4 & NS \\
\hline$(+)$ & 43.1 & & 37.4 & $10 \mathrm{~s}$ & 66.8 & & 28.9 & \\
\hline Therapy after recurre & & & & & & & & \\
\hline $\begin{array}{l}\text { Surgical resection } \\
(-)\end{array}$ & 34.2 & & 32.9 & & 44.0 & & 35.1 & \\
\hline & & $<0.0001$ & & $<0.0001$ & & $<0.0001$ & & $<0.0001$ \\
\hline $\begin{array}{l}\text { Surgical resection } \\
(+)\end{array}$ & 65.8 & & 57.5 & & 74.4 & & 62.2 & \\
\hline
\end{tabular}

CEA: Carcinoembryonic antigen, LN: lymph node, NS: Not significant, Mucinous: mucinous adenocarcinoma, Poorly: poorly differentiated adenocarcinoma, SAR: survival after recurrence, Well/Moderately: well differentiated/moderately differentiated adenocarcinoma.

was an independent prognostic factor for poor SAR in stage III RC patients. Age ( $\geq 75)$, type of recurrence (M1b), and treatment after recurrence (surgical resection) were also identified as independent prognostic factors for SAR in the current study. Surgical resection was considered a strong prognostic factor for advanced CRC $[18,19]$. Recent improvement of treatments against metastatic CRC may provide better SAR than that in current study [20,21].
Therefore, we proposed that patients with prognostic factors for poor SAR should receive intensive treatment and follow-up after curative surgery. These patients include stage III CC patients with undifferentiated adenocarcinoma or N2 nodal status, stage II RC patients with high preoperative CEA level, and stage III RC patients.

Our study had several limitations. First, it was a retrospective study, and we had no genetic information, 
Table 6: Multivariate analysis of prognostic factors for SAR

\begin{tabular}{|c|c|c|c|c|c|c|c|c|}
\hline \multirow{3}{*}{$\begin{array}{l}\text { Location } \\
\text { Stage } \\
\text { Factors }\end{array}$} & \multicolumn{4}{|c|}{ Colon cancer } & \multicolumn{4}{|c|}{ Rectal cancer } \\
\hline & \multicolumn{2}{|c|}{ Stage II } & \multicolumn{2}{|c|}{ Stage III } & \multicolumn{2}{|c|}{ Stage II } & \multicolumn{2}{|c|}{ Stage III } \\
\hline & $\begin{array}{c}\text { HR } \\
\text { CI }\end{array}$ & $P$ & $\begin{array}{c}\text { HR } \\
\text { CI }\end{array}$ & $P$ & $\begin{array}{l}\text { HR } \\
\text { CI }\end{array}$ & $P$ & $\begin{array}{l}\text { HR } \\
\text { CI }\end{array}$ & $P$ \\
\hline $\begin{array}{l}\text { Age: } \\
\quad 75 \leq\end{array}$ & $\begin{array}{c}2.71 \\
1.56- \\
4.67\end{array}$ & 0.0005 & $\begin{array}{c}1.33 \\
0.93-1.9\end{array}$ & NS & $\begin{array}{c}1.77 \\
0.81- \\
3.56\end{array}$ & NS & $\begin{array}{l}1.78 \\
1.03- \\
2.96\end{array}$ & 0.04 \\
\hline $\begin{array}{l}\text { Gender: } \\
\text { Female }\end{array}$ & & & & & & & $\begin{array}{l}1.69 \\
1.14- \\
2.47\end{array}$ & 0.009 \\
\hline $\begin{array}{l}\text { CEA: } \\
\text { High }\end{array}$ & & & & & $\begin{array}{c}2.33 \\
1.34- \\
4.11\end{array}$ & 0.003 & & \\
\hline $\begin{array}{l}\text { Histological type: } \\
\text { Poorly/Mucinous }\end{array}$ & & & $\begin{array}{c}2.04 \\
1.15- \\
3.41\end{array}$ & 0.02 & & & $\begin{array}{c}2.54 \\
1.34- \\
4.54\end{array}$ & 0.005 \\
\hline $\begin{array}{l}\text { Tumor depth: } \\
\text { T4 }\end{array}$ & $\begin{array}{c}1.42 \\
0.84- \\
2.43\end{array}$ & NS & $\begin{array}{c}1.32 \\
0.93- \\
1.87\end{array}$ & NS & & & & \\
\hline $\begin{array}{l}\text { Nodal status: } \\
\quad \text { N2 }\end{array}$ & & & $\begin{array}{c}1.5 \\
1.05- \\
2.12\end{array}$ & 0.03 & & & & \\
\hline $\begin{array}{l}\text { Lymphatic invasion: } \\
\text { ly3 }\end{array}$ & & & $\begin{array}{l}1.23 \\
0.66- \\
2.15\end{array}$ & NS & & & & \\
\hline $\begin{array}{l}\text { Recurrence: } \\
\text { within } 1 \text { year }\end{array}$ & & & & & & & $\begin{array}{c}1.64 \\
1.12-2.4\end{array}$ & 0.01 \\
\hline $\begin{array}{l}\text { Type of recurrence: } \\
\text { M1b }\end{array}$ & $\begin{array}{c}2.50 \\
1.25- \\
4.83\end{array}$ & 0.01 & $\begin{array}{c}1.39 \\
0.84- \\
2.18\end{array}$ & NS & $\begin{array}{c}2.69 \\
1.31- \\
5.25\end{array}$ & 0.008 & $\begin{array}{c}1.68 \\
0.97- \\
2.78\end{array}$ & NS \\
\hline \multicolumn{9}{|l|}{ Recurrence sites } \\
\hline Liver $(+)$ & $\begin{array}{c}1.04 \\
0.58- \\
1.84\end{array}$ & NS & & & & & & \\
\hline Lung: $(+)$ & & & & & $\begin{array}{l}1.25 \\
0.68- \\
2.24\end{array}$ & NS & & \\
\hline Peritoneal: $(+)$ & $\begin{array}{c}0.74 \\
0.35- \\
1.61\end{array}$ & NS & $\begin{array}{l}1.37 \\
0.82- \\
2.36\end{array}$ & NS & & & $\begin{array}{l}1.11 \\
0.53- \\
2.27\end{array}$ & NS \\
\hline Local: $(+)$ & $\begin{array}{c}3.54 \\
1.5-7.69\end{array}$ & 0.005 & & & & & & \\
\hline No adjuvant therapy & & & & & & & $\begin{array}{c}1.00 \\
0.64- \\
1.53\end{array}$ & NS \\
\hline
\end{tabular}




\begin{tabular}{lcccccccc}
\hline Location & \multicolumn{3}{c}{ Colon cancer } & \multicolumn{3}{c}{ Rectal cancer } \\
\hline Stage & \multicolumn{2}{c}{ Stage II } & \multicolumn{2}{c}{ Stage III } & \multicolumn{2}{c}{ Stage II } & \multicolumn{2}{c}{ Stage III } \\
\hline Factors & HR & $\boldsymbol{P}$ & HR & $\boldsymbol{P}$ & HR & $\boldsymbol{P}$ & HR & $\boldsymbol{P}$ \\
& CI & & CI & & CI & & CI & \\
\hline & 5.0 & & 2.36 & & 3.41 & & 4.03 & \\
No surgical resection after recurrence & $2.73-$ & $<0.0001$ & $1.64-$ & $<0.0001$ & $1.93-$ & $<0.0001$ & $2.65-$ & $<0.0001$ \\
& 9.45 & & 3.43 & & 6.18 & & 6.25 \\
\hline
\end{tabular}

CEA: Carcinoembryonic antigen, CI: confidence interval, HR: hazard ratio, Mucinous: mucinous adenocarcinoma, NS: Not significant, Poorly: poorly differentiated adenocarcinoma, SAR: survival after recurrence.

such as RAS mutation and microsatellite instability statuses, which are critical genetic markers for prognosis and treatment. KRAS mutation analysis has been available for clinical use in Japan since 2010. Moreover, universal screening for Lynch syndrome was performed in $<10 \%$ of the hospitals specializing in CRC treatment in Japan [22]. Genetic information was therefore not considered for CRC treatment at the time of surgery in this study. Second, this study also lacked information on the usage of molecular-targeted drugs. Vascular endothelial growth factor and epidermal growth factor antibodies have been used clinically in Japan since 2007 and 2008, respectively. Patients with recurrence should thus have received these drugs for treatment after recurrence. Finally, this was a retrospective study with no rules about follow-up or treatment after surgery, or treatment after recurrence.

Although it seems advisable to detect recurrence as soon as possible to improve the chances of curative resection, the usefulness of intensive follow-up remains controversial. Furthermore, it is unclear if early detection of recurrence could increase the rate of curative treatment and improve survival. Our data demonstrated that short TTR was an independent prognostic factor for SAR in stage III RC patients, who should have received intensive follow-up. However, further prospective studies are needed to confirm our results. Genetic assessment is indispensable in the era of molecular-targeted therapy.

\section{MATERIALS AND METHODS}

\section{Patients and data collection}

This retrospective multicenter study was conducted by the Japanese Study Group for Postoperative Followup of Colorectal Cancer (JFUP-CRC). Clinical data were collected for CRC patients who underwent curative surgery at 22 hospitals in Japan between 2007 and 2008. The study was approved by the institutional review board or ethics committee at each hospital and was performed according to the Declaration of Helsinki and Ethical Guidelines for Clinical Research. All patients provided written informed consent. The JFUP-CRC office pooled and prepared the data available for clinical study, as described in the $7^{\text {th }}$ edition of the Japanese Classification of Colorectal Carcinoma [23]. Lymphatic (ly) or venous (v) invasion was classified according to the degree of invasion, as follows: no invasion (ly $0 / v 0)$, minimal invasion (ly $1 / \mathrm{v} 1)$, moderate invasion (ly $2 / \mathrm{v} 2)$, or severe invasion (ly3/v3). A total of $4992 \mathrm{CRC}$ patients who underwent curative resection in 2007 and 2008, without preoperative chemotherapy/radiotherapy, hereditary $\mathrm{CRC}$, lateral lymph node metastasis, or colitic cancer, were considered suitable for assessment. These patients usually received follow-up with CEA tests every 3 months and CT every 6 months for 3 years, and then CEA every 6 months and CT every 12 months for 3-5 years. The median follow-up time was 72 months in all patients and 60 months in patients with recurrence.

\section{Data analysis}

Differences in clinicopathological factors according to tumor location (CC or $\mathrm{RC}$ ) were analyzed for all patients and for patients with recurrence. Factors associated with recurrence were assessed using $\chi^{2}$ tests.

The influences of clinicopathological factors on relapse-free survival (RFS) and overall survival (OS) in all patients, and TTR and SAR in patients with recurrence were assessed using log-rank tests. Prognostic factors associated with TTR and SAR were subjected to multivariate analyses using a Cox proportional hazard model or logistic analysis. A $P$ value of $<0.05$ was considered significant for all analyses.

\section{CONCLUSIONS}

We recommend that intensive follow-up after surgery is appropriate in stage III CC patients with undifferentiated adenocarcinoma or N2 nodal status, stage II RC patients with high preoperative CEA level, and stage III RC patients.

\section{Abbreviations}

CC: Colon Cancer; CEA: Carcinoembryonic antigen; CRC: Colorectal cancer; CT: Computed tomography; JFUP-CRC: Japanese study group for 
postoperative follow-up of colorectal cancer; NCCN: National Comprehensive Cancer Network; OS; Overall survival; RC: Rectal cancer; RFS; Relapse free survival; SAR: Survival after recurrence; TTR: Time to recurrence.

\section{Author contributions}

Conception and study design: TY. Data analysis: TY, SY. Acquisition of data: TY, SY, KT, MN, MK, MH, AB, KK, CS, AI, ST, MI, NT, KS. Manuscript writing: TY. All authors read and approved the final version of the manuscript.

\section{ACKNOWLEDGMENTS}

We thank the members of JFUP-CRC for collecting the clinical data: I. Takemasa (Sapporo Medical University), K. Hakamada (Hirosaki University), H. Kameyama (Niigata University), Y. Takii (Niigata Cancer Center Hospital), K. Hase (National Defense Medical College), H. Ozawa (Tochigi Cancer Center), H. Nozawa (Tokyo University), K. Takahashi (Tokyo Metropolitan Cancer and Infectious Diseases Center Komagome Hospital), Y. Kanemitsu (National Cancer Center Hospital), M. Itabashi (Tokyo Women's Medical University), H. Yano (National Center for Global Health and Medicine), Y. Kinugasa (Tokyo Medical and Dental University), H. Hasegawa (Keio University), Y. Hashiguchi (Teikyo University), T. Masaki (Kyorin University), M. Watanabe (Kitasato University), T. Hanai (Fujita Health University), K. Komori (Aichi Cancer Center Hospital), Y. Sakai (Kyoto University), M. Ohue (Osaka Medical Center for Cancer and Cardiovascular Diseases), S. Noura (Osaka Rosai Hospital), and Y. Akagi (Kurume University).

We also thank Editage for editing the English text of a draft of this manuscript.

\section{DECLARATIONS}

\section{Ethics approval and consent to participate}

This study was approved by the Central Institutional Review board (Tokyo Medical and Dental University) and local ethical committee. Written informed consent was obtained from all patients.

\section{Availability of data and material}

The datasets used and analyzed during this study are available from the corresponding author on reasonable request under permission of JFUP-CRC.

\section{CONFLICTS OF INTEREST}

The authors declare that they have no conflicts of interest.

\section{REFERENCES}

1. Ferlay J, Soerjomataram I, Dikshit R, Eser S, Mathers C, Rebelo M, Parkin DM, Forman D, Bray F. Cancer incidence and mortality worldwide: sources, methods and major patterns in GLOBOCAN 2012. Int J Cancer. 2015; 136:E359-86.

2. Cancer information service in Japan. Available: http:// ganjoho.jp/public/index.html.

3. Kobayashi H, Mochizuki H, Sugihara K, Morita T, Kotake K, Teramoto T, Kameoka S, Saito Y, Takahashi K, Hase K, Oya M, Maeda K, Hirai T, et al. Characteristics of recurrence and surveillance tools after curative resection for colorectal cancer: a multicenter study. Surgery. 2007; 141:67-75.

4. Verberne CJ, Zhan Z, van den Heuvel E, Grossmann I, Doornbos PM, Havenga K, Manusama E, Klaase J, van der Mijle HC, Lamme B, Bosscha K, Baas P, van Ooijen B, et al. Intensified follow-up in colorectal cancer patients using frequent Carcino-Embryonic Antigen (CEA) measurements and CEA-triggered imaging: results of the randomized "CEAwatch" trial. Eur J Surg Oncol. 2015; 41:1188-96.

5. Pita-Fernández S, Alhayek-Aí M, González-Martín C, López-Calviño B, Seoane-Pillado T, Pértega-Díaz S. Intensive follow-up strategies improve outcomes in nonmetastatic colorectal cancer patients after curative surgery: a systematic review and meta-analysis. Ann Oncol. 2015; 26:644-56.

6. Tjandra JJ, Chan MK. Follow-up after curative resection of colorectal cancer: a meta-analysis. Dis Colon Rectum. 2007; 50:1783-99.

7. Primrose JN, Perera R, Gray A, Rose P, Fuller A, Corkhill A, George S, Mant D, and FACS Trial Investigators. Effect of 3 to 5 years of scheduled CEA and CT follow-up to detect recurrence of colorectal cancer: the FACS randomized clinical trial. JAMA. 2014; 311:263-70.

8. Mokhles S, Macbeth F, Farewell V, Fiorentino F, Williams NR, Younes RN, Takkenberg JJ, Treasure T. Meta-analysis of colorectal cancer follow-up after potentially curative resection. Br J Surg. 2016; 103:1259-68.

9. Jeffery M, Hickey BE, Hider PN, See AM. Follow-up strategies for patients treated for non-metastatic colorectal cancer. Cochrane Database Syst Rev. 2016; 11:CD002200.

10. Watanabe T, Muro K, Ajioka Y, Hashiguchi Y, Ito Y, Saito Y, Hamaguchi T, Ishida H, Ishiguro M, Ishihara S, Kanemitsu Y, Kawano H, Kinugasa Y, et al, and Japanese Society for Cancer of the Colon and Rectum. Japanese Society for Cancer of the Colon and Rectum (JSCCR) Guidelines 2016 for the treatment of colorectal cancer. Int J Clin Oncol. 2018; 23:1-34.

11. Schmoll HJ, Van Cutsem E, Stein A, Valentini V, Glimelius B, Haustermans K, Nordlinger B, van de Velde CJ, Balmana J, Regula J, Nagtegaal ID, Beets-Tan RG, Arnold D, et al. ESMO Consensus Guidelines for management of patients 
with colon and rectal cancer. a personalized approach to clinical decision making. Ann Oncol. 2012; 23:2479-516.

12. Glimelius B, Tiret E, Cervantes A, Arnold D, and ESMO Guidelines Working Group. Rectal cancer: ESMO Clinical Practice Guidelines for diagnosis, treatment and follow-up. Ann Oncol. 2013; 24:vi81-88.

13. Meyerhardt JA, Mangu PB, Flynn PJ, Korde L, Loprinzi CL, Minsky BD, Petrelli NJ, Ryan K, Schrag DH, Wong SL, Benson AB 3rd, and American Society of Clinical Oncology. Follow-up care, surveillance protocol, and secondary prevention measures for survivors of colorectal cancer: American Society of Clinical Oncology clinical practice guideline endorsement. J Clin Oncol. 2013; 31:4465-70.

14. NCCN Clinical Practice Guidelines in Oncology. Colon cancer. Available: https://www.nccn.org/professionals/ physician_gls/pdf/colon.pdf.

15. NCCN Clinical Practice Guidelines in Oncology. Rectal cancer. Available: https:/www.nccn.org/professionals/ physician_gls/pdf/rectal.pdf.

16. Huh JW, Kim CH, Lim SW, Kim HR, Kim YJ. Early recurrence in patients undergoing curative surgery for colorectal cancer: is it a predictor for poor overall survival? Int J Colorectal Dis. 2013; 28:1143-49.

17. Ryuk JP, Choi GS, Park JS, Kim HJ, Park SY, Yoon GS, Jun SH, Kwon YC. Predictive factors and the prognosis of recurrence of colorectal cancer within 2 years after curative resection. Ann Surg Treat Res. 2014; 86:143-51.

18. Hadden WJ, de Reuver PR, Brown K, Mittal A, Samra JS, Hugh TJ. Resection of colorectal liver metastases and extrahepatic disease: a systematic review and proportional metaanalysis of survival outcomes. HPB. 2016; 18:209-20.

19. Leung U, Gönen M, Allen PJ, Kingham TP, DeMatteo RP, Jarnagin WR, D'Angelica MI. Colorectal cancer liver metastases and concurrent extrahepatic disease treated with resection. Ann Surg. 2017; 265:158-65.

20. Loree JM, Kopetz S. Recent developments in the treatment of metastatic colorectal cancer. Ther Adv Med Oncol. 2017; 9:551-64.

21. Tomasello G, Petrelli F, Ghidini M, Russo A, Passalacqua $\mathrm{R}$, Barni S. FOLFOXIRI plus bevacizumab as conversion therapy for patients with initially unresectable metastatic colorectal cancer: a systematic review and pooled analysis. JAMA Oncol. 2017; 3:e170278.

22. Yamano T, Hamanaka M, Babaya A, Kimura K, Kobayashi M, Fukumoto M, Tsukamoto K, Noda M, Matsubara N, Tomita N, Sugihara K. Management strategies in Lynch syndrome and familial adenomatous polyposis: a national healthcare survey in Japan. Cancer Sci. 2017; 108:243-49.

23. Japanese Classification of Colorectal Carcinoma. 7th ed. Tokyo: Kanehara Shuppan; 2009.[in Japanese]. 